\title{
Using Cryo-EM to Map Small Ligands on Dynamic Metabolic Enzymes: Studies with Glutamate Dehydrogenase ${ }^{\mathrm{g}}$
}

\author{
Mario J. Borgnia, Soojay Banerjee, Alan Merk, Doreen Matthies, Alberto Bartesaghi, \\ Prashant Rao, Jason Pierson, Lesley A. Earl, Veronica Falconieri, Sriram Subramaniam, \\ and Jacqueline L. S. Milne
}

Laboratory of Cell Biology, Center for Cancer Research, National Cancer Institute, National Institutes of Health, Bethesda, Maryland (M.J.B., S.B., A.M., D.M., A.B., P.R., L.A.E., V.F., S.S., J.L.S.M.) and FEl Company, Hillsboro, Oregon (J.P.)

Received January 13, 2016; accepted March 31, 2016

\begin{abstract}
Cryo-electron microscopy (cryo-EM) methods are now being used to determine structures at near-atomic resolution and have great promise in molecular pharmacology, especially in the context of mapping the binding of small-molecule ligands to protein complexes that display conformational flexibility. We illustrate this here using glutamate dehydrogenase (GDH), a 336-kDa metabolic enzyme that catalyzes the oxidative deamination of glutamate. Dysregulation of GDH leads to a variety of metabolic and neurologic disorders. Here, we report near-atomic resolution cryo-EM structures, at resolutions ranging from $3.2 \AA$ to $3.6 \AA$ for $\mathrm{GDH}$ complexes,
\end{abstract}

\section{Introduction}

Recent advances in cryo-electron microscopy (cryo-EM) allow determination of structures of small protein complexes and membrane proteins at near-atomic resolution, marking a critical shift in the structural biology field. One specific area of broad general interest in drug discovery is the localization of bound ligands and cofactors under conditions in which efforts at crystallization have not been successful because of structural heterogeneity. Recent cryo-EM analyses have already demonstrated that it is now possible to use single-particle cryo-EM methods to localize small bound ligands or inhibitors on target proteins. Whether ligand binding can be visualized at high resolution is an important question, even in the more general case when multiple conformations are present simultaneously. Here, we address this question using mammalian glutamate dehydrogenase as an example.

This research was supported by funds from the National Institutes of Health National Cancer Institute Center for Cancer Research, the IATAP program at NIH, and the NIH-FEI Living Laboratory for Structural Biology (S.S., J.L.S.M.). This work was supported by the Intramural Research Program of the National Institutes of Health National Cancer Institute.

dx.doi.org/10.1124/mol.116.103382.

[S This article has supplemental material available at molpharm. aspetjournals.org. including complexes for which crystal structures are not available. We show that the binding of the coenzyme NADH alone or in concert with GTP results in a binary mixture in which the enzyme is in either an "open" or "closed" state. Whereas the structure of NADH in the active site is similar between the open and closed states, it is unexpectedly different at the regulatory site. Our studies thus demonstrate that even in instances when there is considerable structural information available from X-ray crystallography, cryo-EM methods can provide useful complementary insights into regulatory mechanisms for dynamic protein complexes.

ABBREVIATIONS: cryo-EM, cryo-electron microscopy; GDH, glutamate dehydrogenase; NBD, nucleotide binding domain 
A

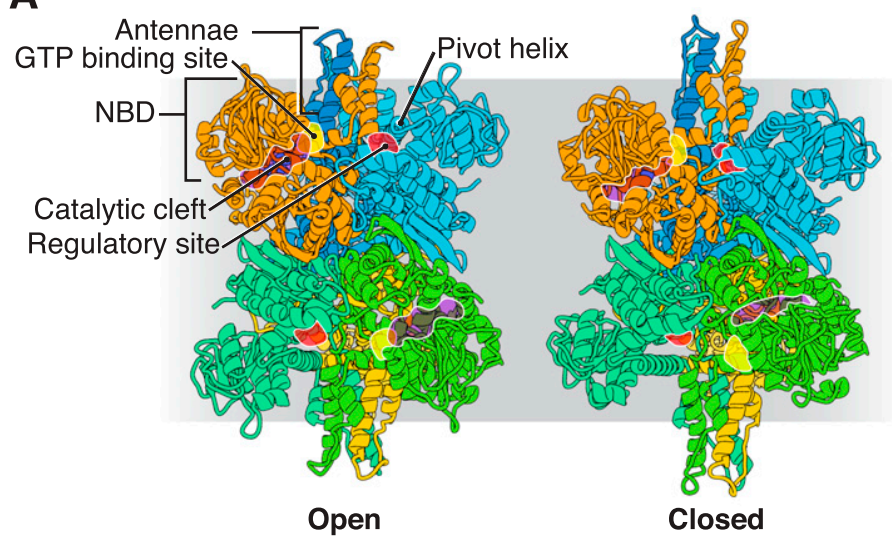

B

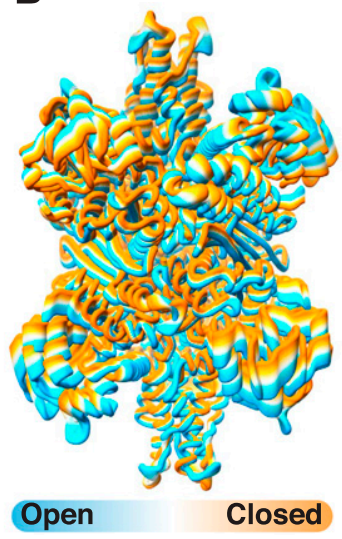

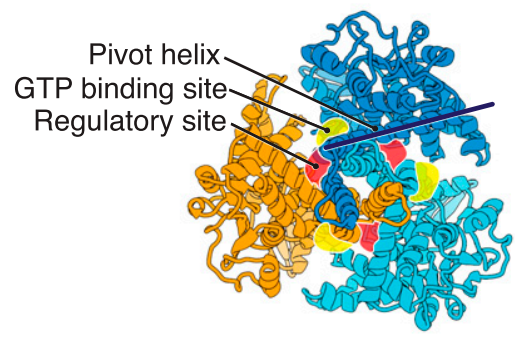
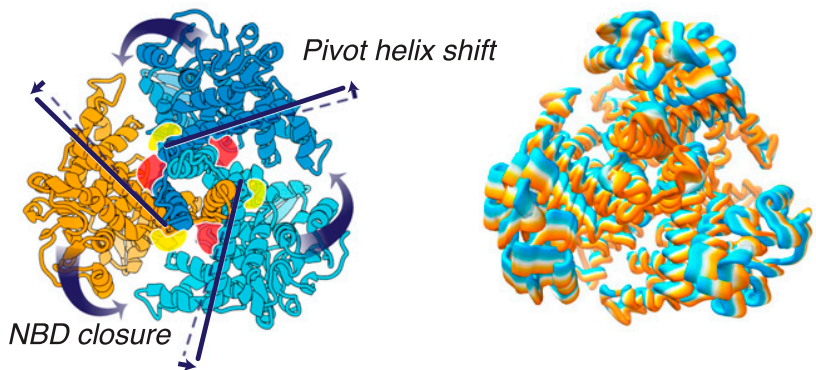

Fig. 1. Structure and quaternary conformational changes in GDH. (A) Views of open (PDB ID 1NR7) and closed (PDB 3MW9) states of the GDH hexamer, shown in ribbon representation perpendicular to the 2 -fold symmetry axis (side view, top) and 3-fold symmetry axis (top view, bottom). Only three protomers are shown in the top view for purposes of visual clarity. The dashed lines and arrows, respectively, highlight the slight extension in length, and twist in shape that occurs with transition from open to the closed state. The open state shown is for unliganded GDH, whereas the closed state has NADH, GTP, and glutamate bound. (B) Superposition of structures for closed and open conformations, along with a series of possible intermediate conformations along the trajectory that serve to illustrate the extent of change in structure across different regions of the protein.

catalytic cleft, and drives a large conformational change in the hexamer from open to closed states (Fig. 1B). The third domain, dubbed the "antenna," is an evolutionary acquisition in protista and animals (Hudson and Daniel, 1993). Antennae of adjacent protomers in each trimer intercalate to form a bundle, perpendicular to the pivot helices, that protrudes along the distal extremes of the 3 -fold axis. When a protomer undergoes a conformational change, the rotation of its pivot helix is transferred through the antenna to the adjacent subunit. The influence of the antenna, present only in protozoan and metazoan enzymes, has been proposed to explain its cooperative behavior, which is absent in bacterial homologs (Smith et al., 2001). Deletion of this domain leads to loss of cooperativity (Allen et al., 2004).

The transition between "closed" and "open" states of GDH is modulated by two allosteric sites in each protomer (Fig. 1A), which are differentially bound by GTP (an inhibitor) and ADP (an activator). These allosteric modulators tightly control GDH function in vivo (Koberstein and Sund, 1973; Bailey et al., 1982; Stanley et al., 1998; Li et al., 2011). In the first site, which sits next to the pivot helix at the base of the antenna (the "GTP binding site"), GTP binding is known to act as an inhibitor, preventing release of the reaction product from the catalytic site by stabilizing the closed conformation of the catalytic cleft (Frieden, 1963; Koberstein and Sund, 1973). In the second "regulatory site", which is situated near the pivot helix between adjacent protomers, ADP acts as an activator of enzymatic activity, presumably by hastening the opening of the catalytic cleft that leads to the release of the reaction product (Koberstein and Sund, 1973; Banerjee et al., 2003). Interestingly, it has also been shown that the coenzyme
$\mathrm{NADH}$ can bind to the regulatory site (also bound by the activator $\mathrm{ADP}$ ), exerting a converse, inhibitory effect on GDH product release, although the role this may play in vivo is not entirely clear (Dieter et al., 1981; Smith et al., 2001; Banerjee et al., 2003).

Although there are numerous crystal structures available for GDH in complex with cofactors and nucleotides, they are limited to the combinations that have been amenable to crystallization. Nearly all X-ray structures of mammalian GDH are in the closed conformation, and the few structures that are in the open conformation are at lower resolution (Table 1). Of those structures in the closed conformation, most include $\mathrm{NAD}[\mathrm{P}] \mathrm{H}, \mathrm{GTP}$, and glutamate (or, alternately, NAD+, GTP, and $\alpha$-ketoglutarate). However, the effects of coenzyme and GTP, bound alone or in concert in the absence of glutamate, have not been analyzed by crystallographic methods. Here, we report single-particle cryo-electron microscopy (cryo-EM) studies that show that under these conditions enzyme complexes coexist in both closed and open conformations. We show that the structures in both states can be resolved at near-atomic resolution, suggesting a molecular mechanism for synergistic inhibition of GDH by NADH and GTP (see Table 2 for detailed information on all cryo-EM-derived structures that we report in this work).

\section{Materials and Methods}

Specimen Preparation. Bovine glutamate dehydrogenase (Enzyme Commission 1.4.1.3; Sigma-Aldrich/MilliporeSigma, St. Louis, MO) was dialyzed overnight against fractionation buffer $(100 \mathrm{mM}$ 
TABLE 1

X-ray structures of mammalian GDH reported in both the open and closed conformations

\begin{tabular}{llllc}
\hline \multicolumn{1}{c}{ GDH } & \multicolumn{1}{c}{ Ligands } & PDB ID & Conformation & Resolution \\
\hline WT & NADH + GLU + GTP & 3MW9 & Closed & 2.4 \\
WT & Glu, GTP, NADPH, and Bithionol & 3ETD & Closed & 2.5 \\
WT & Glu, NADPH, GTP + GW5074 & 3ETG & Closed & 2.5 \\
WT & apo & 1L1F & Open & 2.7 \\
WT & NADPH, glutamate, and GTP & 1HWZ & Closed & 2.8 \\
WT & NADPH + GLU + GTP + Zinc & 3MVQ & Closed & 2.94 \\
WT & NADPH, Glu, GTP, Hexachlorophene & 3ETE & Closed & 3 \\
WT & NAD, PO4, and 2-oxoglutarate & 1HWY & Closed & 3.2 \\
WT & NADPH + GLU + Eu & 3MVO & Closed & 3.23 \\
R463A mutant & apo & 1NR1 & Open & 3.3 \\
WT & apo & 1NR7 & Open & 3.3 \\
WT & ADP & 1NQT & Open & 3.5 \\
WT & NADPH and Epicatechin-3-gallate (Ecg) & 3QMU & Open & 3.62 \\
\hline
\end{tabular}

potassium phosphate, $\mathrm{pH}$ 6.8) prior to fractionation by size-exclusion chromatography using a Superdex 200 10/30 column connected to an ÄKTA FPLC apparatus (GE Healthcare Bio-Sciences, Piscataway, NJ ). The concentration of GDH was adjusted to $\sim 2 \mathrm{mg} / \mathrm{ml}$ by rapid mixing with potassium phosphate buffer containing the concentration of ligand as necessary and with n-octyl glucopyranoside at a final concentration of $0.1 \%$. The final concentration of each ligand was $20 \mathrm{mM}$. Small volumes of sample, typically $3 \mu \mathrm{l}$, were deposited on 200 mesh Quantifoil R2/2 grids (Quantifoil Micro Tools, Großlöbichaum, Germany), blotted, and plunge-frozen in liquid ethane using an FEI Vitrobot Mark IV (FEI Company, Hillsboro, OR). Frozen grids were mounted into autoloader cartridges and transferred to the microscope.

Cryo-Electron Microscopy. Specimens were imaged on an FEI Titan Krios microscope (FEI Company) aligned for parallel illumination and operated at $300 \mathrm{kV}$. The instrument was furnished with a Gatan K2 Summit camera placed at the end of a GIF Quantum energy filter (Gatan Inc., Pleasanton, CA), operated in zero-energy-loss mode with a slit width of $20 \mathrm{eV}$. Images were collected manually at a dose rate of $\sim 5 \mathrm{e}^{-}$pixel $^{-1} \mathrm{~s}^{-1}$, i.e., in the linear range of the detector. The physical pixel size at the plane of the specimen was $1.275 \AA$, corresponding to a super-resolution pixel size of $0.6375 \AA$. The total exposure time was $15.2 \mathrm{~s}$, and intermediate frames were recorded every $0.4 \mathrm{~s}$, giving an accumulated dose of $\sim 45 \mathrm{e}^{-} / \AA^{2}$ and a total of 38 frames per image. The majority of images were collected at under-focus values between $1 \mu \mathrm{m}$ and $3 \mu \mathrm{m}$.

Data Processing. Drift and beam-induced motion were compensated by whole-frame alignment of movies, and CTF was estimated as described in Bartesaghi et al. (2014). Integrated frames were manually examined and selected on the basis of the quality of the CTF estimation, astigmatism, drift, and particle distribution. Molecular images were automatically identified from selected integrated micrographs by detecting the local maxima of correlation of each image with a Gaussian disk of $150 \AA$ in radius. Individual particle projections were extracted from integrated super-resolution images using a binning factor of 4 and a box size of $96 \times 96$ pixels and assigned into $20-100$ groups by iterative reference free two-dimensional classification as implemented in EMAN2 (Tang et al., 2007). Following 8 iterations of classification, a subset of classes depicting intact particles were used to build symmetric (D3) density maps using the program e2initialmodel.py from the EMAN2 suite. One or more maps were selected as reference for further processing on the basis of consistency between projections and the original classes. These "initial models" were refined to $\sim 15-20 \AA ̊$ using e2refine_easy.py. Unbinned particles were then re-extracted from the original super-resolution images using a binning of 2 and a box size of $384 \times 384$, and subject to classification in three dimensions using the maximum likelihood method implemented in RELION (Scheres, 2012; MRC Laboratory, Cambridge, UK) (regularization parameter of $\mathrm{T}=4$ ). Unless noted otherwise, D3 symmetry was imposed for three-dimensional classification runs, the number of classes was initially determined on the basis of the number of particles included in the analysis and later adjusted on the basis of the number of conformations detected in the sample (see Supplemental Table 1 for details). Iteration over classification in three dimensions was continued until convergence as judged by resolution and distribution of particles among the classes. Particles belonging to "good" three-dimensional classes were pooled into one or more classes, depending on the conformational landscape of the complex, and refined using the "gold standard" method in RELION. The refined maps were corrected for the MTF of the camera and for B-factor in the framework of RELION. Figures were generated using UCSF Chimera (Pettersen et al., 2004) and Maxon Cinema4D (Maxon Computer Inc., Newbury Park, CA), and two-dimensionally composited in Adobe Photoshop and Illustrator.

Building of Atomic Models. The deposited models for unliganded GDH (1NR7), the binary complex with ADP (1NQT), and the quaternary complex with NADH, GTP, and Glu (3MW9) were used to derive models from the six structures reported here. Conflicts in sequence between the deposited models were solved by conforming to the primary sequence as reported in 3MW9. The models were placed in the corresponding map (open or closed) by rigid body fitting as implemented in Chimera. In the closed state, in which all relative orientations of the ligands are known, only the ligands known to be present in each structure were retained, all other nonstandard residues were deleted. For the open state, ligands were initially placed on the basis of their orientation relative to the corresponding binding site. The models were refined against a map derived from

TABLE 2

Cryo-EM structures of mammalian GDH determined for this study

\begin{tabular}{clcclcc}
\hline GDH & \multicolumn{1}{c}{ Ligands } & EMDB ID & PDB ID & Conformation & Resolution & Particles \\
\hline WT & apo & EMD-6630 & 3JCZ & Open & 3.26 & 22462 \\
WT & GTP & EMD-6631 & 3JD0 & Open & 3.47 & 39439 \\
WT & NADH & EMD-6635 & 3JD2 & Open & 3.27 & 34716 \\
WT & NADH & EMD-6634 & 3JD1 & Closed & 3.27 & 34926 \\
WT & NADH + GTP & EMD-6632 & 3JD3 & Open & 3.55 & 14793 \\
WT & NADH + GTP & EMD-6633 & 3JD4 & Closed & 3.40 & 20429 \\
\hline
\end{tabular}


one-half of the dataset using Rosetta as described (https://faculty. washington.edu/dimaio/files/density_tutorial.pdf), followed by real space refinement in PHENIX (Adams et al., 2010; PHENIX, Berkeley, $\mathrm{CA}$ ) and evaluated by calculating the Fourier shell correlation between the model and the map derived from the second half of the dataset. For each complex, ten best scoring instances on the basis of the Fourier shell correlation were selected among one hundred runs, visually examined, and the one deemed best interactively corrected in Coot (Emsley et al., 2010). Each corrected model was subject to a final instance of real space refinement using PHENIX.

\section{Results and Discussion}

To explore the conformational landscape of apo-GDH, we first determined its structure in the absence of any added ligands (Supplemental Fig. 1, Fig. 2, A-C). The density map, refined to an average resolution of $\sim 3.0 \AA$ (Supplemental Fig. 2 ), is in the open conformation and closely matches the model of unliganded GDH derived by X-ray crystallography at $3.3 \AA$ resolution (PDB ID 1NR7). The variation in local resolution from the core to the periphery, as reported by ResMap (Kucukelbir et al., 2014) (Supplemental Fig. 3D), is consistent with the B-factor gradient observed in the crystal structure (Supplemental Fig. 3A). Extensive classification without imposing symmetry yielded only open structures and failed to detect any closed catalytic cleft in the unliganded enzyme, suggesting that all six protomers are in the open conformation. Consistent with this conclusion, the loops connecting the $\beta$-strands of the Rossmann fold are well-defined (Fig. 2B), implying that there is little movement at the NBD, as the transition between closed and open states is associated with NBD movement (Fig. 1B).

When GDH is bound to NADH, GTP, and glutamate, the enzyme adopts a closed conformation; this "abortive complex" has been determined to $2.4-\AA$ resolution by X-ray crystallography (PDB 3MW9). However, crystal structures of GDH bound only to NADH or to GTP have not yet been reported. To test the effect of NADH binding on GDH conformation in solution, we determined the structure of this binary complex using cryo-EM methods combined with three-dimensional classification. Two dominant conformational states, in an all open or all closed conformation were detected, segregated (Fig. 2D), and further refined to near-atomic resolution $(\sim 3.3 \AA$;
A

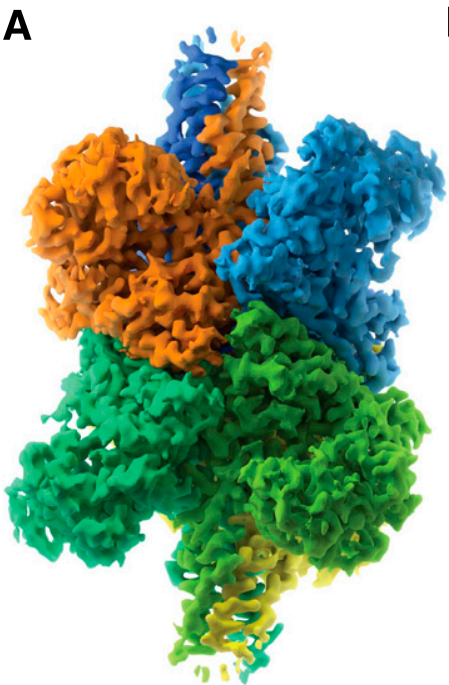

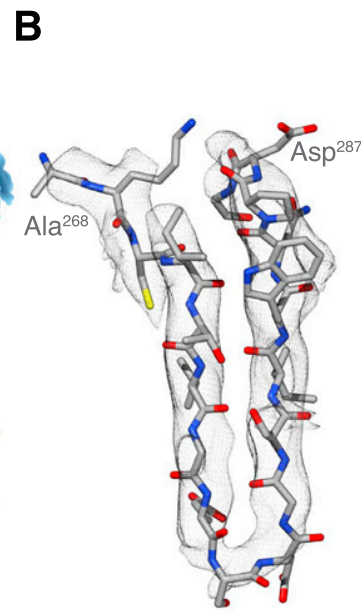

Rossmann fold segment, NBD

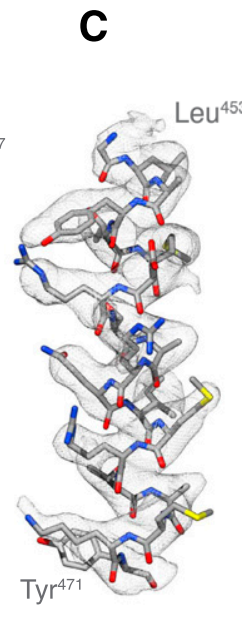

Pivot helix

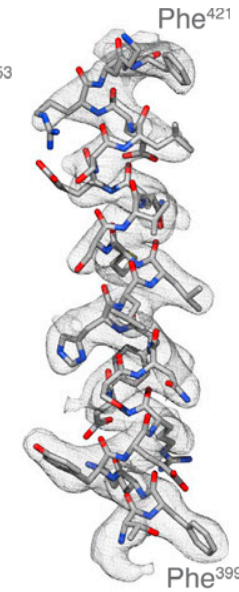

Antenna
D

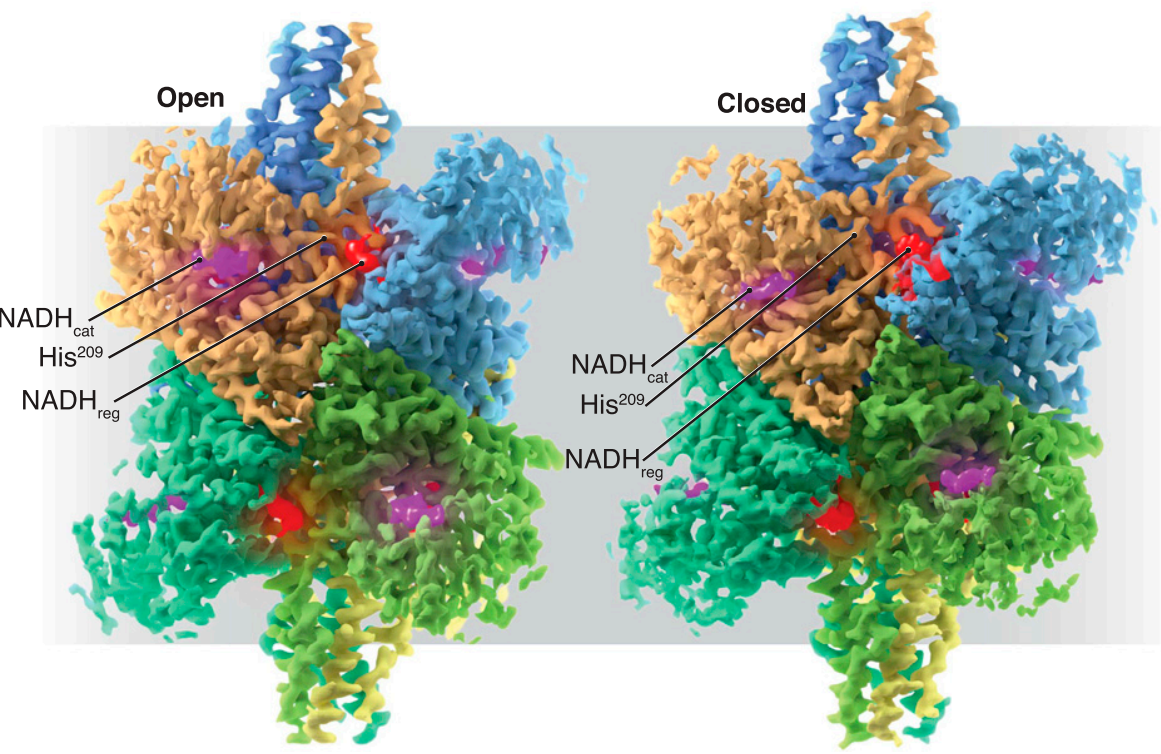

Fig. 2. Cryo-EM structures of GDH in unliganded and NADH-bound states. (A) Refined cryo-EM map of unliganded GDH at $\sim 3$ A resolution. (B, C) Illustration of density map in the regions that contain the Rossmann nucleotide binding fold (B), pivot and antenna helices (C) in the unliganded GDH map. (D) CryoEM-derived density maps for two coexisting conformations that are present when GDH is bound to the cofactor $\mathrm{NADH}$. Each protomer is shown in a different color and densities for NADH bound in both regulatory (red) and catalytic (purple) sites on one protomer are indicated. The overall quaternary structures of the two conformations are essentially the same as that of the open and closed states observed by X-ray crystallography. 
Supplemental Fig. 2). Densities for 12 molecules of bound NADH were identified in maps of both open and closed states (Supplemental Fig. 4). The NADH-bound closed conformation matches the structure of the quaternary complex observed by $\mathrm{X}$-ray crystallography, with the exception that density corresponding to GTP and glutamate was absent in the cryo-EMderived map.

Comparison of the NADH-bound closed conformation to the $\mathrm{NADH}$-bound open conformation shows that, as expected, the catalytic cleft is closed and the NBDs are displaced toward the equatorial plane, accompanied by a rotation of the pivot helix by $\sim 7^{\circ}$, concomitant with a large conformational change in the antennae domains (Figs. 1 and 2D). A comparison between NADH-bound open and closed conformations also involves a displacement of helix 5 (residues 171-186), as well as a tilt of the core $\beta$-sheets relative to the equatorial plane of the enzyme (residues 57-97, 122-130) and $\alpha$-helix 2 (residues 36-54), and a bending of the N-terminal helix. Thus, closure of the catalytic cleft is accompanied by a quaternary structural change that can be described as a global bending of the structure about an axis that runs parallel to the pivot helix, accompanied by an expansion of the core (Figs. 1A and 2D).

Detailed analysis of the GDH/NADH structures shows that both the adenosine and nicotinamide moieties of $\mathrm{NADH}$ bind to the catalytic site within the NBD in nearly the same orientation in both the open and the closed states, and display closely comparable interactions with the Rossmann fold (Fig. $3, \mathrm{~A}$ and $\mathrm{B}$ ). At the regulatory site, where either ADP can bind as an activator or NADH can bind as an inhibitor (Koberstein and Sund, 1973; Bailey et al., 1982; Banerjee et al., 2003), the binding of the adenine moiety of $\mathrm{NADH}$ is nearly identical between the two conformers. However, there is a significant difference in the orientation of the nicotinamide and phosphate moieties in the two conformational states (Fig. 3, C and D). In the closed state, the nicotinamide group is oriented toward the center of the hexamer, inserted into a narrow cavity between two adjacent subunits in the trimer. There are extensive interactions between $\mathrm{NADH}$ and the residues lining this cavity, which may explain the well-defined density of this portion of $\mathrm{NADH}$ in the closed state. In contrast, in the open conformation, the cavity present in the closed state becomes too narrow for the nicotinamide group; instead, the group is oriented in the opposite direction, parallel to the pivot helix with the amido group extending toward the C-terminal end of the helix.

Although there is a difference in orientation of the nicotinamide moiety between the closed and open states in the regulatory site, in both structures the adenine portion of $\mathrm{NADH}$ has a similar binding pocket and is located in almost exactly the same position as ADP, a potent activator of GDH function (Supplemental Fig. 5). In the open state, the binding of $\mathrm{ADP}$ or $\mathrm{NADH}$ is further stabilized by $\mathrm{His}^{209}$, a residue that undergoes a large movement during the transition from open to closed conformation (Fig. 3, C and D). In the open conformation, the distance between $\mathrm{His}^{209}$ and the $\alpha$-phosphate of NADH is $\sim 4.4 \AA$, which is comparable with the corresponding distance in the ADP-bound conformation (Banerjee et al., 2003). In the closed conformation, however, this key histidine residue is $>10.5$ $\AA$ away from the nearest phosphate group on $\mathrm{NADH}$, altering a critical stabilization point within the regulatory site. This suggests that although the conformation of $\mathrm{NADH}$ in the open state regulatory site more closely mimics the binding of $\mathrm{ADP}$, the conformation of $\mathrm{NADH}$ in the closed state regulatory site is significantly different; these differences may contribute to the opposite effects of NADH and ADP on GDH enzymatic activity.

In the absence of NADH, GTP binds weakly to GDH with a dissociation constant of $\sim 20 \mu \mathrm{M}$ (Frieden, 1965; Koberstein and Sund, 1973). Cryo-EM analysis of GDH incubated with GTP resulted in a structure at an overall resolution of $3.5 \AA$, showing that it is in an open conformation (Supplemental Fig. 6),
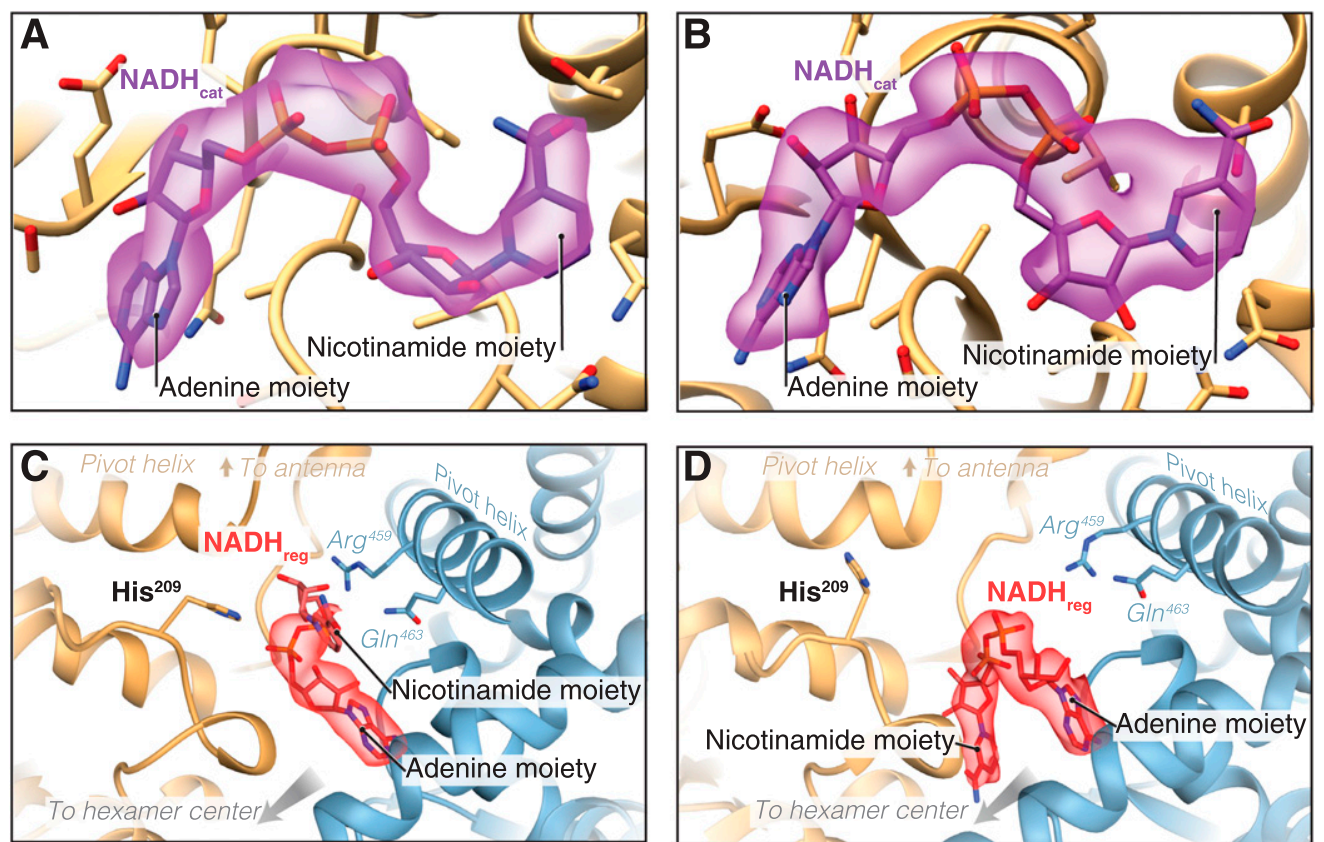

Fig. 3. Detailed view of NADH conformation in catalytic and regulatory sites. (A, B) NADH density (purple) and interactions in the catalytic sites of closed (A) and open (B) states. (C, D) NADH density (red) and interactions in the regulatory sites of closed (C) and open (D) states. 
with all NBDs in the open state. The density for GTP is not very well defined, suggesting considerable wobble in the binding site. Subtraction of the GTP-bound map with that of the apo state shows that GTP binding can nevertheless be visualized specifically in the GTP binding site (Supplemental Fig. 6). Importantly, the binding of GTP alone does not appear to drive the transition from the open to the closed state of GDH.

To further dissect the roles of NADH and GTP in the transition from the open to closed conformations, we next determined structures of GDH in complex with both NADH and GTP, but without glutamate. When NADH and GTP are both present, classification reveals the presence of both closed and open GDH conformations, similar to the condition when only $\mathrm{NADH}$ is present (Fig. 4, A and B). Reconstruction without classification, however, yields a structure clearly in the closed conformation, suggesting that, in coordination with $\mathrm{NADH}$, GTP may further stabilize the closed conformation. The location of GTP in the open and closed states of the GDH/NADH/GTP complex is similar to that in the crystal structure observed in the presence of $\mathrm{NADH}$, GTP, and glutamate (Smith et al., 2001). Likewise, the position of $\mathrm{NADH}$ in the open and closed states closely resembles the position of $\mathrm{NADH}$ in the GDH/NADH open and closed structures. One key difference between the open and closed states of these structures is the position of the $\mathrm{His}^{209}$ residue: As mentioned above, His ${ }^{209}$ swings away from the adenine moiety of NADH in the closed state. When GTP is present in the GTP binding site, $\mathrm{His}^{209}$ instead interacts with GTP, probably stabilizing the closed conformation (Fig. 4, C and D). Thus, GTP binding to GDH appears synergistic with $\mathrm{NADH}$ and displaces the conformational landscape toward the closed state.

Our structural studies thus establish that whether or not GTP is bound, NADH binding is detectable at catalytic and regulatory sites, in both the open and closed conformational states. Whereas the orientation in which $\mathrm{NADH}$ binds at the catalytic site is similar for both conformations, the orientation of the nicotinamide portion of $\mathrm{NADH}$ in the regulatory site is different between the open and closed conformations (Figs. 3 and 4). In the closed state, the nicotinamide moiety is inserted into a well-defined cavity at the interface between two adjacent protomers in the trimer. As mentioned above, this cavity is much narrower in the open state, suggesting that this cavity may be unavailable to the $\mathrm{NADH}$ nicotinamide moiety when the enzyme is in the open conformation. These structural features provide a potential explanation of the weaker density for the nicotinamide moiety of $\mathrm{NADH}$ in the open state, and may account for the higher reported affinity of NADH for the closed state (Shafer et al., 1972; Koberstein and Sund, 1973). The role of the nicotinamide moiety in acting as a wedge that prevents the transition to the open conformation also suggests a structural explanation of the mechanism by which $\mathrm{NADH}$ binding inhibits the activity of the enzyme by stabilizing the closed conformation state.

The rapid emergence of cryo-EM as a tool for near-atomic resolution structure determination provides new opportunities for complementing atomic resolution information from X-ray crystallography, as illustrated here with GDH. Perhaps the most important contribution of these methods is the prospect that when there are discrete subpopulations present, the structure of each state can be determined at near-atomic resolution. What we demonstrate here with $\mathrm{GDH}$ is that by employing three-dimensional image classification approaches, we not only can isolate distinct, coexisting conformations, but we can also localize small molecule ligands in each of these conformations. These kinds of approaches will probably become increasingly important in molecular pharmacology, especially

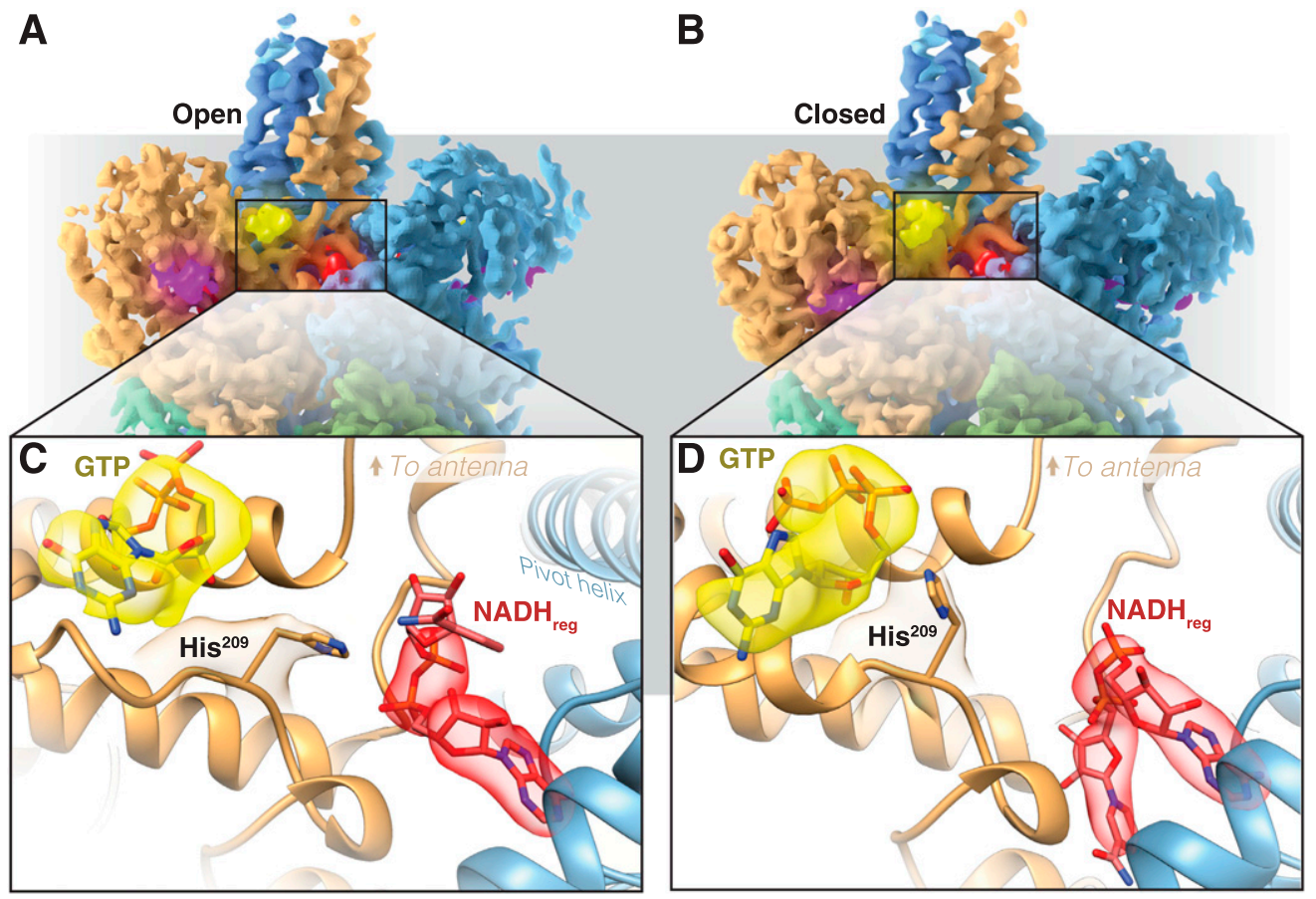

Fig. 4. Cryo-EM structure of GDH bound to both NADH and GTP. (A, B) Observation of co-existing open (A) and closed (B) conformations in the GDHNADH-GTP ternary complex. Densities for GTP (yellow) as well as NADH bound to both catalytic (purple) and regulatory (red) sites in each protomer are shown. (C, D) Detailed inspection of the interactions near the regulatory site show that the orientation of His ${ }^{209}$ switches between the two states, which may allow interactions with bound GTP in the closed (D), but not open (C) conformation. 
in the context of better understanding drug-target interactions in dynamic protein complexes.

\section{Acknowledgments}

This work used the computational resources of the NIH HPC Biowulf cluster (http://hpc.nih.gov). The density maps and refined atomic models have been deposited with the Electron Microscopy Data Bank (accession numbers EMD-6630, EMD-6631, EMD-6632, EMD6633, EMD-6634, EMD-6635) and Protein Data Bank (entry codes PDB3JCZ，PDB-3JD0，PDB-3JD3，PDB-3JD4，PDB-3JD1，PDB-3JD2), respectively.

\section{Authorship Contributions}

Participated in research design: Borgnia, Banerjee, Merk, Subramaniam, Milne.

Conducted experiments: Borgnia, Banerjee, Merk, Rao, Pierson.

Performed data analysis: Borgnia, Banerjee, Merk, Matthies, Bartesaghi, Earl, Falconieri, Subramaniam, Milne.

Wrote or contributed to the writing of the manuscript: Borgnia, Banerjee, Earl, Falconieri, Subramaniam, Milne.

\section{References}

Adams PD, Afonine PV, Bunkóczi G, Chen VB, Davis IW, Echols N, Headd JJ, Hung LW, Kapral GJ, and Grosse-Kunstleve RW et al. (2010) PHENIX: a comprehensive Python-based system for macromolecular structure solution. Acta Crystallogr D Biol Crystallogr 66:213-221.

Allen A, Kwagh J, Fang J, Stanley CA, and Smith TJ (2004) Evolution of glutamate dehydrogenase regulation of insulin homeostasis is an example of molecular exaptation. Biochemistry 43:14431-14443.

Bailey J, Bell ET, and Bell JE (1982) Regulation of bovine glutamate dehydrogenase. The effects of $\mathrm{pH}$ and ADP. J Biol Chem 257:5579-5583.

Banerjee S, Schmidt T, Fang J, Stanley CA, and Smith TJ (2003) Structural studie on ADP activation of mammalian glutamate dehydrogenase and the evolution of regulation. Biochemistry 42:3446-3456.

Bartesaghi A, Matthies D, Banerjee S, Merk A, and Subramaniam S (2014) Structure of $\beta$-galactosidase at $3.2-\AA$ - resolution obtained by cryo-electron microscopy. Proc Natl Acad Sci USA 111:11709-11714.

Couée I and Tipton KF (1990) Inhibition of ox brain glutamate dehydrogenase by perphenazine. Biochem Pharmacol 39:1167-1173.

Dieter H, Koberstein R, and Sund H (1981) Studies of glutamate dehydrogenase. The interaction of $\mathrm{ADP}, \mathrm{GTP}$, and $\mathrm{NADPH}$ in complexes with glutamate dehydrogenase. Eur J Biochem 115:217-226.

Emsley P, Lohkamp B, Scott WG, and Cowtan K (2010) Features and development of Coot. Acta Crystallogr D Biol Crystallogr 66:486-501.
Frieden C (1963) Glutamate Dehydrogenase. V. The relation of enzyme structure to the catalytic function. $J$ Biol Chem 238:3286-3299.

Frieden C (1965) Glutamate Dehydrogenase. VI. Survey of purine nucleotide and other effects on the enzyme from various sources. J Biol Chem 240:2028-2035.

George A and Bell JE (1980) Effects of adenosine 5'-diphosphate on bovine glutamate dehydrogenase: diethyl pyrocarbonate modification. Biochemistry 19:6057-6061.

Hudson RC and Daniel RM (1993) L-glutamate dehydrogenases: distribution, properties and mechanism. Comp Biochem Physiol B 106:767-792.

Koberstein R and Sund H (1973) Studies of glutamate dehydrogenase. The influence of ADP, GTP, and L-glutamate on the binding of the reduced coenzyme to beef-liver glutamate dehydrogenase. Eur J Biochem 36:545-552.

Kucukelbir A, Sigworth FJ, and Tagare HD (2014) Quantifying the local resolution of cryo-EM density maps. Nat Methods 11:63-65.

Li C, Li M, Chen P, Narayan S, Matschinsky FM, Bennett MJ, Stanley CA, and Smith TJ (2011) Green tea polyphenols control dysregulated glutamate dehydrogenase in transgenic mice by hijacking the ADP activation site. J Biol Chem 286:34164-34174.

Li M, Li C, Allen A, Stanley CA, and Smith TJ (2012) The structure and allosteric regulation of mammalian glutamate dehydrogenase. Arch Biochem Biophys 519: 69-80.

Pettersen EF, Goddard TD, Huang CC, Couch GS, Greenblatt DM, Meng EC, and Ferrin TE (2004) UCSF Chimera-a visualization system for exploratory research and analysis. J Comput Chem 25:1605-1612.

Rife JE and Cleland WW (1980) Kinetic mechanism of glutamate dehydrogenase. Biochemistry 19:2321-2328.

Scheres SH (2012) RELION: implementation of a Bayesian approach to cryo-EM structure determination. J Struct Biol 180:519-530.

Shafer JA, Chiancone E, Vittorelli LM, Spagnuolo C, Mackler B, and Antonini E (1972) Binding of reduced cofactor to glutamate dehydrogenase. Eur J Biochem $\mathbf{3 1}$ $166-171$.

Smith TJ, Peterson PE, Schmidt T, Fang J, and Stanley CA (2001) Structures of bovine glutamate dehydrogenase complexes elucidate the mechanism of purine regulation. J Mol Biol 307:707-720.

Stanley CA, Lieu YK, Hsu BY, Burlina AB, Greenberg CR, Hopwood NJ, Perlman K, Rich BH, Zammarchi E, and Poncz M (1998) Hyperinsulinism and hyperammonemia in infants with regulatory mutations of the glutamate dehydrogenase gene. N Engl J Med 338:1352-1357.

Tang G, Peng L, Baldwin PR, Mann DS, Jiang W, Rees I, and Ludtke SJ (2007) EMAN2: an extensible image processing suite for electron microscopy. J Struct Biol 157:38-46.

Tomkins GM, Yielding KL, and Curran JF (1962) The influence of diethylstilbestrol and adenosine diphosphate on pyridine nucleotide coenzyme binding by glutamic dehydrogenase. J Biol Chem 237:1704-1708.

Yielding KL and Tomkins GM (1961) An effect of L-leucine and other essential amino acids on the structure and activity of glutamic dehydrogenase. Proc Natl Acad Sci USA 47:983-989.

Address correspondence to: Dr. Sriram Subramaniam and Dr. Jacqueline L. S. Milne, 50 South Drive, Rm 4306, MSC 8008, Bethesda, MD 20892. E-mail: ss1@nih.gov and jmilne@mail.nih.gov 